\title{
Regarding "Mystery of Chronic Cerebrospinal Venous Insufficiency: Identical Venographic and Ultrasound Findings in Patients with MS and Controls"
}

W e would like to comment on the recently published study entitled "Mystery of Chronic Cerebrospinal Venous Insufficiency: Identical Venographic and Ultrasound Findings in $\mathrm{Pa}-$ tients with MS and Controls" by McAuliffe and Kermode. ${ }^{1}$

The study aimed to analyze a very interesting and controversial topic that recently produced much debate in medical and patients' circles. In our opinion, to achieve their goal, the authors used questionable design and methodology, which resulted in numerous limitations of their study. ${ }^{1}$

Namely, the limited number of the patients and control subjects included makes the whole series inconclusive. Data were missing about the training of actual investigators concerning chronic cerebrospinal venous insufficiency (CCSVI), keeping in mind that the patients examined were their initial 30 cases. ${ }^{1}$

The authors found internal jugular vein narrowing in 8 of 9 patients without MS by use of venography, but they did not perform sonography (ultrasound) examination before catheterization to determine if these patients might have CCSVI without MS. Therefore, they did not confirm or exclude the presence of CCSVI by ultrasound examination in these patients. Venography performed in these patients only describes the similarity of internal jugular vein narrowing seen in patients with CCSVI and other pathologies. Similarly, by use of ultrasound examination, the authors found no case of CCSVI in the MS group of patients or healthy control subjects, but they did not confirm the absence of CCSVI with catheter venography. ${ }^{1}$

We do not know if MS is connected with CCSVI, yet morphologic and hemodynamic abnormalities of jugular veins have been seen in patients with MS as well. ${ }^{2}$

Considering that CCSVI is a new entity and has yet to be de- fined, besides the ultrasound, we have used MDCT angiography to register and evaluate extracranial venous pathway obstruction in patients with MS. ${ }^{3}$ MDCT proved to be a very reliable procedure for extracranial venous intraluminal obstruction and extraluminal compression diagnosis. ${ }^{3}$

In addition to MDCT, during venous percutaneous angioplasty we measured gradient pressures before and after the venoplasty at various obstruction levels to confirm hemodynamic significance of the diagnosed lesions. ${ }^{3}$

Whether in patients with MS or healthy control subjects, ultrasound, MDCT, or catheter venography and gradient pressure measurement should be performed before the final diagnosis of CCSVI can be made.

Finally, multicentric prospective studies with long-term follow-up are necessary to resolve numerous questions concerning CCSVI that many patients with MS and their physicians pose daily.

\section{REFERENCES}

1. McAuliffe W, Kermode AG. Mystery of chronic cerebrospinal venous insufficiency: identical venographic and ultrasound findings in patients with MS and controls. AJNR Am J Neuroradiol January 31, 2013. [Epub ahead of print]

2. Radak DJ, Kolar J, Tanaskovic S, et al. Morphological and haemodynamic abnormalities in the jugular veins of patients with multiple sclerosis. Phlebology 2012;27:168-72

3. Radak D, Tanaskovic S, Antonic Z, et al. Compressive syndrome of internal jugular veins in multiple sclerosis: does it matter? Phlebology September 7, 2012. [Epub ahead of print]

D.J. Radak N. Ilijevski S. Tanaskovic "Dedinje" Cardiovascular Institute Vascular Surgery Clinic School of Medicine, Belgrade University Belgrade, Serbia 